\title{
Enhancer-silencer transitions in the human genome
}

Di Huang and Ivan Ovcharenko*

Computational Biology Branch, National Center for Biotechnology Information, National

Library of Medicine, National Institutes of Health, Bethesda, MD, 20892, USA.

* To whom correspondence should be addressed: ovcharen@ nih.gov

\section{Running title}

Enhancer-silencer transitions

\section{Key words}

deep learning, dual-function regulatory element, enhancers, silencers, transcription factor binding sites 


\begin{abstract}
Dual-function regulatory elements (REs), acting as enhancers in some cellular contexts and as silencers in others, have been reported to facilitate the precise gene regulatory response to developmental signals in Drosophila melanogaster. However, with few isolated examples detected, dual-function REs in mammals have yet to be systematically studied. We herein investigated this class of REs in the human genome and profiled their activity across multiple cell types. Focusing on enhancer-silencer transitions specific to the development of $\mathrm{T}$ cells, we built an accurate deep learning classifier of regulatory elements and identified about 12,000 silencers active in primary peripheral blood $\mathrm{T}$ cells that act as enhancers in embryonic stem cells. As compared to regular silencers, these dual-function REs are evolving under stronger purifying selection and are enriched for mutations associated with disease phenotypes and altered gene expression. In addition, they are enriched in the loci of transcriptional regulators, such as transcription factors (TFs) and chromatin remodeling genes. Dual-function REs consist of two intertwined but largely distinct sets of binding sites bound by either activating or repressing TFs -- depending on the type of RE function in a given cell line. This indicates the recruitment of different TFs for different regulatory modes and a complex DNA sequence composition of these REs with dual activating and repressive encoding. With an estimated over $6 \%$ of cell-typespecific human silencers acting as dual-function REs, this overlooked class of REs requires a specific investigation on how their inherent functional plasticity might be a contributing factor to human diseases.
\end{abstract}

\title{
Introduction
}


Transcriptional silencers, exerting negative regulatory impact and counterbalancing positive regulatory elements (REs, such as enhancers), have long been recognized to be essential for fine-tuning gene regulation and precisely responding to cellular signals and environmental stimuli in metazoans (Jacob and Monod 1961; Brand et al. 1985; Johnson et al. 2015; Rojano et al. 2018; Halfon 2020). However, due to the inherent difficulty in assaying and identifying silencers, the research focused on silencers has been greatly overshadowed by studies targeting enhancers. With the scarcity of known examples, knowledge about silencers has lagged behind that of enhancers, promoters, and even that of insulators (Ngan et al. 2020). Recently, the advance of massively parallel reporter assays (MPRAs) has greatly facilitated the large-scale detection of silencers in human cells and ignited interest in silencers (Della Rosa and Spivakov 2020; Doni Jayavelu et al. 2020; Pang and Snyder 2020).

Despite recent progress, large-scale silencer maps are still restricted to very few cell lines in humans, specifically HepG2 liver carcinoma and K562 chronic myelogenous leukemia cell lines (Doni Jayavelu et al. 2020; Pang and Snyder 2020). This only offers a limited characterization of the effects of silencers in various biological contexts. Furthermore, while histone $\mathrm{H} 3$ lysine 27 acetylation (H3K27ac) and histone H3 lysine 4 mono-methylation (H3K4me1) have been widely used for enhancer identification, the most promising mark for silencer identification, histone $\mathrm{H} 3$ lysine 27 trimethylation (H3K27me3), is not specific enough to accurately separate silencers from enhancers (Della Rosa and Spivakov 2020; Gisselbrecht et al. 2020). In human embryonic stem cells (hESCs), almost two-thirds of H3K27me3 chromatin immunoprecipitation sequencing (ChIP-seq) peaks carry signals of the histone marks associated with transcriptional activation. In primary T cells, a differentiated cell type, half of the H3K27me3 ChIP-seq peaks exhibit the same property. Furthermore, there is a lack of 
consistency among silencer maps reported by different groups. In the HepG2 cell line for example, two independent sets of silencers, which were identified using massively parallel platforms and contain $\sim 4,000$ elements each, have only four silencers in common (Doni Jayavelu et al. 2020; Pang and Snyder 2020). This small overlap could be partially explained by different silencer pooling strategies and suggests an abundance of silencers active in a human cell type. Inspired by the success of sequence-based deep learning models in the prediction of chromatin states, TF binding occupancies, and enhancers (Alipanahi et al. 2015; Zhou and Troyanskaya 2015; Thibodeau et al. 2018) as well as our prior work on silencer detection (Huang et al. 2019), we trained a sequence-based deep learning model to identify silencers and demonstrated that our model greatly outperforms other methods for silencer detection.

After generating accurate genome-wide maps of cell-specific silencers, we decided to focus on dual-function REs (DFREs), which act either as silencers or enhancers in different cell lines and thus represent the versatile component of gene regulatory machinery. Limited DFREs have been reported in Drosophila melanogaster (Stathopoulos and Levine 2005) and mice (Bessis et al. 1997; Kehayova et al. 2011). More recently, DFRE abundance in D. melanogaster has been reported (Erceg et al. 2017; Gisselbrecht et al. 2020). Almost 25\% of nearly 1,000 Pho Recessive Complex (PhoRC)-bound silencers in mesodermal cells function as enhancers at other developmental stages (Erceg et al. 2017), while in another study, 22 out of 29 (76\%) identified mesodermal silencers act as DFREs (Gisselbrecht et al. 2020). Although the estimates of the fraction of silencers acting as DFREs differed between these two studies, likely since different silencer subclasses were investigated, both studies clearly demonstrated that DFREs are widespread, at least in D. melanogaster embryonic cells. In this study, we focused on human DFREs, investigating distinct functional characteristics of these elements in comparison to 
regular silencers/enhancers and molecular mechanisms enabling their functional transition across cellular contexts.

\section{Results}

\section{Convolutional neural network model accurately predicts silencers}

We built a multi-class convolutional neural network $(\mathrm{CNN})$ model with three nodes in the output layer representing silencers, enhancers, and regulatory neutral DNA sequences (Fig. 1A, see Methods). Our CNN model achieved a respectable prediction performance for silencer and enhancer identification across multiple human cell types. The area under the curve of the receiver operating characteristic (AUC-ROC) ranged from 0.84 to 0.94 for enhancer detection and from 0.74 to 0.90 for silencer detection across six cell types (H1 hESC, hematopoietic progenitor cells (HPC), HepG2, K562, monocyte, and T cell, Figs. 1B and S1).

To perform an independent assessment of the accuracy of silencer predictions, we applied our CNN model to experimentally identified silencers. On two sets of silencers active in K562 cells (see Methods), our CNN model achieved the performance of AUC-ROC $\geq 0.78$ and AUCprecision recall curve (AUC-PRC) $\geq 0.47$ (Fig. 1C). It outperforms the linear support vector machine (SVM) model we developed previously (13) (AUC-ROC $\geq 0.66$ and AUC-PRC $=0.32$ ). The improved accuracy of the CNN model in comparison to the SVM model can be attributed to the superiority of CNNs in capturing spatial patterns in sequences and retrieving nonlinear connections among these patterns (Alipanahi et al. 2015; Zhou and Troyanskaya 2015; Koo and Ploenzke 2020). It is important to note that our method accurately predicts experimentally identified ReSE (Pang and Snyder 2020) and MPRA (Doni Jayavelu et al. 2020) silencers (AUC ROC = 0.81 and 0.78 for ReSE and MPRA silencers, respectively, Fig. 1C). 
Despite a small overlap between these two datasets (less than $0.2 \%$ of matching sequences), the silencers from these datasets share common DNA sequence motifs, such as the enrichment in TFBSs of the repressive TFs REST and SETDB1 (Fig. S2). Silencers predicted by the CNN model also feature similar TFBS enrichment (Fig. S2), indicative of this model capturing the motifs shared across different silencer sets and demonstrating a reliable performance on two otherwise distinct silencer sets. There are also differences in the TFBS enrichment profiles of these three silencer sets, which could reflect different types of silencers within them.

To profile human silencers, we applied the trained CNN model to all DNA sequences carrying open chromatin signals (i.e., DNase-ChIP peaks) or repressive histone marks (i.e., H3K27me3 ChIP-seq peaks) and labelled the sequences having a silencer prediction score larger enough to correspond a less-than- 0.1 false positive rate (FPR) on test samples as silencers (Methods). We also applied this scheme to identify enhancers. To this end, we predicted approximately 130,000 silencers and 120,000 enhancers per cell line (Table S1 and Fig. S3). Among the false positive silencer predictions, i.e., those that carry no H3K27me3 signals but are predicted as silencers, $7.7 \%$ correspond to enhancer candidates, i.e., the DNase-seq peaks having H3K27ac but no H3K27me3 signals in the corresponding cell type. The remaining 92.3\% of false positive silencer predictions correspond to the background, i.e., genomic regions with no H3K27ac, H3K27me3, or DNase signals in the corresponding cell type. In comparison to the test sample sets in which 5.6\% are enhancer candidates (Fig. S4), these results suggest a bias of false positive silencer predictions to enhancer candidates. This likely reflects shared sequence motifs of enhancers and insulators active in a particular cell type that correspond to the binding sites of multi-functional TFs (Berest et al. 2019). For example, the NFKB2 complex functions as both activator and repressor in T cells (Senftleben et al. 2001; Grinberg-Bleyer et al. 2018), and its 
TFBSs are enriched in both T cell silencers and enhancers (see the section "Distinct sequence syntax of the DFREs").

In a systematic high-resolution activation and repression profiling with MPRA (SharprMPRA), the activating/repressive capabilities of individual nucleotides within regulatory elements have been measured by MaxPos scores (Ernst et al. 2016). A low/high MaxPos score indicates a strong repressive/activating regulatory effect. In K562 cells, predicted silencers show the lowest average MaxPos score among all elements, which is 4.7 times lower than the average MaxPos score of H3K27me3 ChIP-seq peaks not predicted as silencers (dubbed nonSLs, Student's $t$-test $p=4 \times 10^{-6}$, Fig. 1D). Similarly, predicted enhancers display a higher average MaxPos score than other elements, including H3K27ac ChIP-seq peaks not predicted as enhancers (dubbed nonEN, Student's $t$-test $p=10^{-22}$, Fig. 1D). These experimental results provide an independent support of our silencer and enhancer predictions.

In the five tested cell lines (monocytes were not included in this analysis due to the lack of gene expression data for this cell type), the predicted silencers were enriched in the proximity of low-expression genes (Fig. S5). Additionally, the density of single nucleotide polymorphisms (SNPs) detected in genome-wide association studies (GWASs) is greater in predicted silencers and enhancers than in the sequences carrying H3K27me3 but no H3K27ac ChIP-seq peaks for all examined cell lines but hESC (binomial test, $p<10^{-10}$, Fig. 1E). Furthermore, we observed that silencers show higher genomic mappability than $\mathrm{H} 3 \mathrm{~K} 27 \mathrm{me} 3$ regions overall (Fig. S6). To exclude a potential ascertainment bias arising from mappability differences between different sequence sets, we restricted the GWAS SNP analysis to the elements with at least $50 \%$ of certainly mappable nucleotides (i.e., mappability score $=1$, Fig. S7). As there is no qualitative change in the results, this further confirms the functional importance of the predicted silencers, at 
least in comparison to $\mathrm{H} 3 \mathrm{~K} 27 \mathrm{me} 3$ regions and randomly selected background sequences with matching length, GC content, and repeat density. For simplicity, we refer to these predicted silencers/enhancers as silencers/enhancers for the remainder of the manuscript.

\section{DFREs are associated with TFs and chromatin remodeling genes}

In our search for REs capable of alternating between enhancer and silencer function, we focused on $\mathrm{H} 1 \mathrm{hESC}$ enhancers that transition to silencers in primary $\mathrm{T}$ cells, a type of differentiated lymphoid cells. Based on overlap with H1 hESC enhancers, the silencers in primary T cells have been split into DFRE and non-DFRE silencers (see Methods). The latter were termed regular silencers (or SLrs). To address the function of DFREs, we compared DFREs with SLrs and hESC enhancers that do not function as enhancers or silencers in T cells (named as ENrs below).

In primary T cells, 11,888 silencers (6\% of all silencers) are DFREs (Fig. 2A). While DFREs and SLrs show similar H3K27me3 intensities (Fig. S8), eight percent of these DFRE sequences are conserved across placental species (Siepel et al. 2005), which is significantly higher than $4.1 \%$ of SLrs and 3.6\% of the background (binomial test $p<10^{-10}$ ) but lower than $10.6 \%$ of ENrs $\left(p<10^{-10}\right.$, Fig. 2B). Also, the DFREs harbor 5.06 common SNPs per kb, which is significantly lower than 5.44 common SNPs per $\mathrm{kb}$ in SLrs $\left(p<10^{-10}\right)$ and is similar to that of ENrs (Fig. 2B). In addition, 7.96\% of DFRE SNPs have a derived allele frequency (DAF) of greater than 0.9, which is significantly lower than 9.23\% of the SLr SNPs $\left(p<10^{-10}\right.$, Fig. S9). These results suggest that DFREs have been and are still evolving under strong purifying selection, which is indicative of their functional importance. 
Next, we used genes flanking DFREs to assess their potential function. As compared to all silencers, the DFREs show a strong preference for the loci of genes participating in transcriptional regulation (such as mRNA transcription, hypergeometric test $p=2 \times 10^{-8}$, Fig. $\mathrm{S} 10$ ) and/or genes associated with binding activity (such as T cell receptor binding, $p=3 \times$ $10^{-5}$, Fig. 2C). Overall, $19.2 \%$ of DFREs are located in the loci of genes encoding TFs and/or chromatin modifiers (CMs), which is significantly higher than $16.7 \%$ of SLrs (binomial test $p=10^{-11}$, Fig. 2D, see Methods). This trend strengthens in the loci of the top 1,000 highexpression TFs and/or CMs by 1.3 times ( $p=6 \times 10^{-5}$, high-expression versus all TFs/CMs). Furthermore, as compared to ENrs, DFREs show a similar enrichment in the loci of all TFs/CMs ( $p=0.03$ ) but a contrasting strong preference to TFs/CMs highly expressed in T cells (binomial test $p=10^{-7}$, Fig. 2D), suggesting a distinct association of DFREs with the regulation of the TFs/CMs specific to T cells. The TFs are the core of gene regulation networks, and CM enzymes, which reshape the chromatin topologies globally or locally to facilitate or impede the binding of TFs, are crucial for regulating the responses to cellular and external signals, especially during cell differentiation and in the context of immunity (Dixon et al. 2015; Tartey and Takeuchi 2015; Chen et al. 2020). The strong association of DFREs with these genes suggests a primary contribution of DFREs to governing the activity and identity of T cells.

Next, we utilized RNA-seq gene expression (Roadmap Epigenomics Consortium et al. 2015) and Hi-C contact data in hESC and T cells (Jung et al. 2019; Yang et al. 2020) to address if a transition of DFRE function might result in change of the DFRE target gene. Among intergenic DFREs that contact only one of their flanking genes (named A) and not the other flanking gene (named X) in hESCs, we identified DFREs that also feature an expression level of A at least five-fold greater than the corresponding $\mathrm{X}$ and the average gene expression level in 
hESC. This set of DFRESs thus constituted the most pronounced DFRE enhancer effects on a specific target gene in hESCs. We then observed that $84.6 \%$ of these A genes in T cells are expressed at a less than two-fold greater level than both the corresponding $\mathrm{X}$ genes and the average gene expression in T cells, and $100 \%$ of these A genes are expressed at a less than fivefold greater level than the corresponding $\mathrm{X}$ genes and the average gene expression in $\mathrm{T}$ cells. This indicates a pronounced drop in the level of A gene expression upon DFRE transition consistent with the enhancer-silencer DFRE change with no change in the target gene. Therefore, these results strongly suggest that the target gene of DFREs remains largely unchanged upon enhancer to silencer transition. In addition, we identified nine intergenic DFREs targeting a single flanking gene in both hESC and T cells. All (8/8) of these single target gene contacts remain intact upon DFRE transition from being an enhancer in hESCs to becoming a silencer in T cells (Fig. S11 for two examples), further supporting preservation of DFRE targets posttransition. These particular target genes include well-known T cell-differentiation regulators TPD52 (Kang et al. 2021) and CHD7 (Koh et al. 2015). The lack of change in chromatin contacts to proximal genes further highlights that the function transition of DFREs is pivotal for precisely regulating the transcription of these $\mathrm{T}$ cell differentiation genes during the development of T cells.

\section{DFREs are enriched in the $\mathbf{T}$ cell-related genetic variants}

To evaluate the influence of DFREs, we next explored GWAS SNPs and their linkagedisequilibrium (LD) counterparts $\left(r^{2}>0.8\right.$, see Methods). The DFREs and SLrs harbor 0.93 and 0.91 GWAS SNPs per kb, respectively, which represents a significant enrichment over 0.74 GWAS SNPs per kb in the background sequences (i.e., randomly sampled genomic sequences 
with the length, GC content, and repetitive element density matching T cell silencers; binormal test $p<10^{-10}$, Fig. 2E). Furthermore, 10.6\% of GWAS SNPs in DFREs are associated with immunity-related traits and $9.8 \%$ of SLr SNPs are immunity-associated. Both percentages are higher than $8.5 \%$ of ENr SNPs, and $9.4 \%$ of background SNPs ( $p<10^{-4}$, Fig. 2E), highlighting the contribution of these silencers, especially DFREs ( $p=0.0002$, DFREs versus SLrs), to the regulation of the immune system. Analyzing individual GWAS traits, we observed that T cell DFREs are specifically enriched in SNPs linked to immune system disorders, such as polyangiitis-granulomatosis, Sjogren syndrome, and type I diabetes mellitus (binormal test $p<0.05$, Fig. 2F and Table S2). Eighteen out of the top fifty DFRE-associated GWAS traits $(36 \%)$ are immunity-related (as indicated by azure diamonds in Fig. 2F), which is significant given that there are about $8 \%$ of immunity-related traits among all GWAS traits (hypergeometric test $\left.p=10^{-7}\right)$

In addition, T cell DFREs host 0.98 whole-blood expression quantitative trait loci (eQTLs) per kb, which is significantly higher than 0.91 in the SLrs, 0.89 in ENrs, and 0.8 in the background sequences (binormal test $p<10^{-5}$, Fig. 2E). Furthermore, the density of T cell expression quantitative loci (eQTLs) in the DFREs is 0.012 per $\mathrm{kb}$, which is 1.5 times that in SLrs $\left(p=2 \times 10^{-6}\right)$ and 1.7 times that in ENrs $\left(p=2 \times 10^{-8}\right)$ and the background sequences $\left(p=9 \times 10^{-9}\right)$. As compared to whole blood eQTLs, T cell eQTLs exhibit elevated enrichment in the T cell silencers, especially the DFREs (chi-squared test $p<10^{-5}$, Fig. 2E), suggesting strong tissue-specificity and pronounced regulatory impact of these silencers on target gene expression in T cells. Also, the DFREs harbor 0.009 eQTLs detected in induced pluripotent stem cells (iPSCs) per kb, which is similar to that in ENrs (binomial test $p=0.13$ ) but is 1.7 times that in SLrs $\left(p=2 \times 10^{-7}\right)$ and 1.4 times that in the background ( $\left.p=0.002\right)$. Combined, the 
enrichment in immunity-associated SNPs and stem-cell-associated SNPs supports the functional duality of the DRFEs, a distinct feature among all tested elements.

\section{DFRE mutations are more likely to negatively affect silencer activity than SLr mutations}

To further characterize the effects of silencer mutations, we compared the output of our CNN models for wild-type alleles to those for mutant alleles, defining the CNN-based silencing alteration score (dubbed CNN-SAS, see Methods and Fig. S12). A large positive value of CNNSAS suggests that the corresponding mutation causes a strong decrease in repressive activity. To evaluate CNN-SAS scores, we first turned to the experimental results in Reporter Assay Quantitative Trait Loci (namely, raQTL) studies where the regulatory effect of a SNP mutation was quantified by the expression change of the reporter gene (van Arensbergen et al. 2019). Positive raQTL scores represent mutations causing a decrease in reporter gene expression. Using the HepG2 CNN model, we calculated the CNN-SASs of HepG2 raQTL mutations and observed that the absolute values of the CNN-SASs of raQTLs were significantly higher than those of non-raQTLs (Wilcoxon rank-sum test $p=4 \times 10^{-164}$, Figs. 3A and S13, see Methods). We also observed a gradual decrease in raQTL scores with an increase in CNN-SASs (Fig. 3B). Among silencer raQTLs with the 5\%-highest CNN-SAS scores, 94\% had negative raQTL scores, which is a significant enrichment given that $54 \%$ of all tested raQTLs had negative raQTL scores (binomial test $p=3 \times 10^{-25}$, Fig. 3B). The fraction of negative raQTLs dwindled with the decrease of CNN-SASs, ending at $12 \%$ among the raQTLs with the 5\%-lowest CNN-SASs ( $p=10^{-25}$ as compared to the expected on all tested raQTLs). The strong negative correlation between CNN-SAS and raQTL scores validates the ability of our method to quantify the strength of silencer-disrupting mutations. 
In T cells, for which raQTL data is not available, we used eQTL data to evaluate CNNSASs. T cell CNN-SAS scores are correlated with T cell eQTLs (Spearman's correlation $r=0.064, p=0.008$, Fig. 3C), potentially confirming the ability of CNN-SAS scores to predict the regulatory impact of mutations in T cells. The weak correlation between CNN-SASs and whole blood eQTLs $(r=0.003, p=0.35$, Fig. S14) could be attributed to the indirect measurement of regulatory activity using eQTLs, as eQTLs reflect association and not causality of noncoding mutations with gene expression due to a) linkage-disequilibrium in loci containing regulatory variants and b) a cumulative effect of multiple regulatory elements on target gene expression (as opposed to a direct readout of single mutation effects in raQTL experiments).

We next used the T cell CNN-SASs to assess the impact of silencer mutations. A CNNSAS was considered significant when its absolute value was larger than $1 \%$ of those of all possible single-nucleotide mutations in the silencers (Fig. S15, Methods). As compared to the ENrs and background sequences, DFREs and SLrs host more significant CNN-SASs among GWAS SNPs and eQTLs (binomial test $p \leq 0.002$ ), reflecting the functional importance of the DFREs and SLrs to the regulation of T cell phenotypes and gene expression. In addition, as compared to SLrs, the DFREs are enriched for significant CNN-SASs (i.e., potential damaging mutations) across a panel of mutation sets (binomial test $p<10^{-5}$, Fig. 3D). For example, in DFREs, 3.5\% of T cell eQTLs SNPs correspond to a significant CNN-SAS (that is 3.5-fold greater than expected by chance; $p<10^{-10}$ ). These results validate the utility of significant CNN-SASs in detecting most likely silencer-damaging mutations.

An example DFRE mutation having a significant CNN-SAS is rs12631656. Its T-to-C (i.e., major to minor allele) mutation corresponds to a CNN-SAS of $0.0816(p=0.003$, Fig. 3E). This SNP is located within a tight LD block $\left(r^{2}>0.96\right)$ of rs6445975 and rs4681851, the two SNPs 
that have been reported to be significantly associated with systemic lupus erythematosus and systemic sclerosis (Harley et al. 2008; Mayes et al. 2014). This LD block hosts five DFRE SNPs, four SLr SNPs, and one enhancer SNP in T cells. Among these SNPs, rs12631656 is the only one with a significant CNN-SAS (Fig. 3E). The TF motif mapping result shows that the mutation at rs12631656 potentially weakens the binding affinity of ARID5B and SOX13, two known repressors in T cells (Lefebvre 2010; Wang et al. 2020).

\section{Distinct sequence syntax of the DFREs}

To address function encryption in DFRE sequences, we considered two primary hypotheses: 1) there is a single set of TF binding sites (TFBSs) within each DFREs bound by a stable set of TFs with alternating repressor and activator functions, and 2) there are two distinct sets of TFBSs within DFREs--one encoding silencer function and another encoding enhancer function. To test these hypotheses, we mapped the TFBSs in TF ChIP-seq peaks ("TFBS prediction in TF ChIPseq peaks" in Supplementary Notes). With these TFBSs, we first characterized TFBS signatures of the DFREs for their activating and repressive functions. In T cells, while inactive ENrs are depleted of ChIP-seq TFBSs of all TFs, silencers (DFREs and SLrs) are depleted of ChIP-seq TFBSs of activators (such as JUND, IRF3 and IRF4) but are enriched in ChIP-seq TFBSs of ubiquitous repressors, including REST and repressors acting predominantly in blood cells, such as EBF1 (Györy et al. 2012) (Fig. 4A), which is in line with their repressive function.

Functioning as enhancers in hESCs, the DFREs and ENrs show a similar TFBS profile. They are depleted of ChIP-seq TFBSs of REST $(p \leq 0.02)$ but are enriched in ChIP-seq TFBSs of hESCspecific TFs, such as POU5F1 and NANOG (Fig. 4B). These results suggest that DFREs recruit a distinct set of TFs for activating and repressive functions. Furthermore, $70 \%$ of silencer TFBSs 
are silencer-specific, and $45 \%$ of enhancer TFBSs are enhancer-specific (Fig. 4C), indicating a presence of distinct DFRE fragments that establish activating and repressive functions.

The enrichment of CTCF ChIP-seq TFBSs in DFREs prompted us to compare DFREs and insulators. In hESCs, where the topologically associated domains (TADs) have been reported (Liu et al. 2019), 0.84\% of the DFREs are located at the TAD boundaries which are known to be enriched for insulators (Ong and Corces 2014). This fraction is comparable to $0.94 \%$ of all DNase-seq peaks $(p=0.25)$ and is significantly lower than $1.12 \%$ of CTCF ChIP-seq TFBSs $\left(p=10^{-11}\right.$, Fig. S16A). These trends were also observed in T cells, where $0.95 \%$ of DFREs, $0.93 \%$ of all DNase-seq peaks ( $p=0.34$ versus DFREs), and $1.31 \%$ of CTCF ChIP-seq TFBSs ( $p=10^{-11}$ versus DFREs, Fig. S16B) are located within the T cell TADs detected in Yang's study (Yang et al. 2020). Also, in T cells, DFREs are enriched in the loci of the 1,000 lowest expressed genes $(9.1 \%$ of DFREs versus $6.3 \%$ of the whole genome, binomial test $p=5 \times$ $10^{-20}$, Fig. S16C) and are depleted in the loci of the 1,000 highest expressed genes $(p=2 \times$ $\left.10^{-5}\right)$. A reverse trend was observed for DFREs in hESCs $(p \leq 0.001$, Fig. S16D). Similarly, the DFREs frequently target the lowly expressed genes in T cells ( $p=10^{-6}$, Fig. S16E) but highly expressed genes in hESCs ( $p=4 \times 10^{-15}$, Fig. S16F). These results are consistent with the silencer function of DFREs in T cells and the enhancer function of DFREs in hESCs. On the other hand, CTCF ChIP-seq TFBSs show no consistent correlation with either highly or lowly expressed genes in both cell types (Fig. S16), supporting a functional distinction between DFREs and CTCF-defined insulators.

We also observed that the majority of DFRE TFBSs, specifically $60 \%$ of enhancer TFBSs and $62 \%$ of silencer TFBSs, are located within the central component $( \pm 200 \mathrm{bp}$ from the midpoint) of the DFREs, which is significantly greater than $43 \%$ of randomly scattered TFBSs 
(binomial test $p<10^{-10}$, Fig 4D). Furthermore, 37\% of the silencer TFBSs are located within 50 bps to their nearest enhancer TFBSs, which is much higher than $24 \%$ of randomly scattered TFBSs in DFREs (binomial test $p=10^{-160}$, Fig. 4E). Combined, these advocate for intertwined and spatially proximal distribution of distinct activating and repressive TFBSs within DFREs.

Next, to account for TFs not yet profiled in ChIP-seq experiments, we used CNN-SASs to predict TFBSs as short segments overrepresented in high CNN-SASs ("TFBS prediction using a CNN model" in Supplementary Notes). More than $43 \%$ of the CNN-predicted TFBSs overlap the reported TF ChIP-seq peaks in both T cells and hESCs, which is over 1.4 times that of randomly scattered TFBSs (Fig. S17A), and therefore justifies the use of this predictive approach for additional TFBS discovery. Predicted TFBSs in T cell (silencer) DFREs are enriched with the binding motifs of repressors, such as SNAI1/2, REST and LMO2 (Fig. S17B). Predicted TFBSs in hESC (enhancer) DFREs host represent binding motifs of developmental activators, including POU5F1, NANOG and SOX4 (Fig. S17C). The CNN-predicted TFBSs confirm and further strengthen the trends observed using ChIP-seq TF data. Fifty-nine percent of these predicted TFBSs reside within the central region $( \pm 200 \mathrm{bp}$ from the midpoint) of DFREs, which is significantly more than the expected $45 \%$ of randomly scattered TFBSs (binomial test $p=$ $\left.10^{-323}\right)$. Also, $43 \%$ of silencer TFBSs are located within $50 \mathrm{bp}$ of enhancer TFBSs, which is significantly more than the expected $32 \%$ of randomly scattered TFBSs $\left(p=10^{-323}\right.$, Fig. S17). Again, these results suggest a tight intertwining between TFBSs active for opposite functions within DFREs as well as a "co-encryption" within the central regions of DFREs.

\section{Developmental dynamics of DFRE formation}


To understand the progression in DFRE formation during development, we applied our analysis pipelines for identifying DFREs in primary HPCs, which represent an intermediate step in differentiation from embryonic stem cells to T cells. Twenty-seven percent of HPC silencers are DFREs, acting as enhancers in hESCs (Fig. 5A). As compared to HPC SLrs, these DFREs are more conserved across placental species (10.2\% of DFREs versus $7.4 \%$ of SLrs, binomial test $\left.p<10^{-10}\right)$ and feature a lower density of common SNPs (4.93 SNPs/kb in DFREs versus 5.45 SNPs/kb in the SLrs, $p<10^{-10}$, Fig. 5B). DFREs also harbor 0.98 GWAS SNPs and 1.3 whole blood eQTLs per kb, which is significantly higher than 0.87 GWAS SNPs and 0.94 whole blood eQTLs in the SLrs, 0.91 GWAS SNPs and 0.96 whole blood eQTLs in the ENrs (i.e., the hESC enhancers acting neither as silencers nor as enhancers in HPCs), as well as 0.74 GWAS SNPs and 0.8 whole blood eQTLs in the background sequences $\left(p<10^{-5}\right.$, Fig. 5C). DFREs are more highly enriched in GWAS and eQTL SNPs than the SLrs, ENrs, and background in both tested cell lines, suggesting their functional importance across cellular contexts. Overall, HPC DFREs feature functional characteristics very similar to T cell DFREs, with the main exception of approximately four times as many HPC silencers being DFREs as compared to T cell silencers (binomial test $p<10^{-5}$ ). Furthermore, among 18,965 HPC DFREs, $11.6 \%(2,201)$ are the DFREs in T cells, which is significantly lower than $18.6 \%$ of HPC SLrs and $46.5 \%$ of HPC enhancers shared by T cells (binomial test $p<10^{-5}$, Fig. 5D). The shrinking fraction of DFREs during differentiation and the strong cell-specificity of DFREs in a mature cell suggest the loss of functional plasticity in regulatory elements upon differentiation and a relatively small fraction of regulatory elements preserving multi-functional activity after the differentiation.

\section{Discussion}


Advances in high-throughput sequencing and massively parallel reporter assays vastly increase the knowledge of gene regulation in human cells. However, the detection and functional characterization of active silencers remain challenging (Della Rosa and Spivakov 2020; Halfon 2020). In this study, we developed a deep learning silencer model, which accurately detects experimentally identified silencers and quantifies the impact of silencer mutations. Identified silencers predominantly populate the loci of low-expression genes and are enriched in GWAS SNPs associated with the cell types matching disease etiology. In principle, silencers exert the repressive impact mainly through reducing the activity of basal promoters or smothering the activity of proximal enhancers (Gisselbrecht et al. 2020). H3K27me3 modification has been evidenced to take part in both repressive mechanisms (Ogiyama et al. 2018; Cai et al. 2021). As such, the CNN models, which were built with H3K27me3 ChIP-seq peaks as training silencer samples, predict both types of silencers. These predicted silencers can be further categorized by utilizing chromatin interaction maps with the knowledge that the silencers that reduce promoter activity interact with promoters more frequently than the other silencers. Also, our models centered on the H3K27me3-associated silencers, might be less accurate in identifying silencers not marked by H3K27me3. Further studies are needed for investigate the differences and similarities between H3K27me-based and H3K27me3-independent silencers.

By combining silencer detection with enhancer detection, we observed enhancer-silencer transitions and found that $6 \%$ of the T cell silencers and $28 \%$ of HPC silencers are DFREs, functioning as enhancers in hESCs and changing their regulatory function during differentiation. As compared to regular silencers, DFREs feature greater evolutionary sequence conservation and are enriched in GWAS SNPs and eQTLs. Moreover, DFREs are preferentially distributed in the proximity of genes governing transcriptional regulation. DFRE mutations are $>1.5$ times as 
likely to significantly damage silencer activity (as measured using CNN-SASs) as regular silencer mutations. These results support the essentiality and a rather overlooked important role of DFREs in regulating the development and maintaining the fate of blood cell types (and likely, other differentiated cell types not included in this study). Earlier studies in Drosophila melanogaster embryonic cells, coupled with the scope of our study targeting a single differentiation path, suggest that a large fraction of developmental enhancers might be wired to act as silencers upon and after differentiation (Erceg et al. 2017; Gisselbrecht et al. 2020).

To gain insights into the mechanisms of DFRE functional duality, we compared the distribution of active TFBSs corresponding to silencer and enhancer functions of DFRE sequences. We observed largely distinct but proximal DFRE regions occupied by binding sites of opposing functions. Our findings suggest a "double" genomic regulatory encryption within DFRE sequences with independent active regulatory binding sites, reflective of the use of different TFs for establishing activating and repressive functions after effectively rejecting an alternative hypothesis of the same pool of TFs bound to DFREs and switching their function based on cellular context.

Silencers are an integral part of the regulatory machinery in metazoans shaping and finetuning gene regulatory programs. The difference in the function of DFREs reported in Drosophila melanogaster was primarily attributed to different cell types. In our study, we focus on the functional switch from enhancer to silencer activity, which is associated with the development and maturation of T cells. Therefore, it is logical to assume that the presented DFREs are more likely to be associated with developmental abnormalities and carcinogenesis. It is also possible that a subset of reported DFREs might underlie the environmental response, and their mutations are more likely to have a phenotypic effect due to their role in development. Here 
we report thousands of DFREs during development of T cells and uncover unique features and profound phenotypic/pathogenic contributions of these elements. These findings together suggest the widespread of DFREs in various biological processes across species. Follow-up studies extending to other biological contexts and additional chromatin data will further characterize human DFREs and understand how the same parts of the genome are reused for multiple regulatory functions at different developmental time points and in different cellular contexts. This is a crucial step toward revealing how gene regulation swiftly and precisely adapts to changing of cellular contexts in various biological processes.

\section{Methods}

\section{Building CNN models to predict silencers}

We designed a deep learning model composed sequentially of five convolutional layers and two fully connected network layers (Fig. 1A). Each CNN layer is followed by a max-pooling and a dropout layer. The input of this model is $1,000 \mathrm{bp}$-long DNA sequences, while the output layer contains three nodes, each representing one of the sequence classes: silencer, enhancer, and background. Each output node predicts the probability of a given sequence belonging to the corresponding sequence classes. We utilized the Python library Keras version 2.4.0 (https://github.com/keras-team/keras) to implement our model. To deal with this multi-class classification task, categorical cross-entropy was used as the cost function. By minimizing this cost function, the parameters of the models were adjusted by using the gradient-based algorithm Adam and then Adagrad as implemented in Keras ("CNN model" in Supplementary Notes and Fig. S18). 
We built a CNN model for each of six cell types, including H1 hESC, K562 cell line, HepG2 cell line, primary hematopoietic progenitor cells (HPC), primary monocyte, and primary T cell from peripheral blood. During training, we set aside Chromosome 6 for validation and Chromosomes 7 and 8 for testing. All other autosomes and Chromosome $\mathrm{X}$ were used for training.

To identify silencers, we calculated the cutoff in the silencer prediction scores (silencer scores) generated by the CNN model, which corresponds to the false positive rate (FPR) of 0.1 in test samples among which background samples were randomly selected so that the ratio of background samples to enhancers/silencers was 9:1 in each tested cell type. Sequences that carry a DNase-seq peak or a $\mathrm{H} 3 \mathrm{~K} 27 \mathrm{me} 3 \mathrm{ChIP}$-seq peak and have a silencer score greater than this cutoff were labelled as silencers. A similar approach with the FPR of 0.1 in CNN model enhancer prediction scores (enhancer scores) was used for enhancer identification. DNase-seq peaks that carry a $\mathrm{H} 3 \mathrm{~K} 27 \mathrm{ac}$ signal and have an enhancer score greater than this cutoff established the set of predicted enhancers. After applying this scheme to all tested cell types, we identified approximately 130,000 silencers and 120,000 enhancers per cell type (Table S1). The size of these enhancer sets is comparable to those identified by ChromHMM (Ernst and Kellis 2017) as well as those collected in Benton's and Gao's databases (Benton et al. 2019; Gao and Qian 2020).

\section{Data for training and validating CNN models}

We trained a CNN enhancer/silencer model independently for each cell type. Cell-type specific DNase-seq and histone modification ChIP-seq datasets have been used for training (all ChIP-seq data used in this study were downloaded from the NIH Roadmap Epigenomics Project (Roadmap Epigenomics Consortium et al. 2015)). H3K27me3 ChIP-seq peaks with neither 
H3K27ac nor H3K4me1/3 ChIP-seq peaks overlapping their 400 bp central region constituted the set of training silencers. DNase-seq peaks with an overlapping H3K27ac peak but no overlapping H3K27me3 ChIP-seq peaks within their 400 bp central region constituted the set of training enhancers. Background sequences were randomly selected from DNase-seq peaks and H3K4me1, H3K4me3, H3K27ac, and H3K27me3 histone modification ChIP-seq peaks detected in any cell line other than the tested cell line. CNN models were trained on $1 \mathrm{~kb}$ regions centered on enhancer, silencer and background sequences. For T cells, we collected 416,773 DNase-seq peaks and 392,442 H3K27me3 and 129,500 H3K27ac "broad" peaks from the NIH Roadmap Epigenomics Project (Roadmap Epigenomics Consortium et al. 2015). This translated into 239,925 and 158,887 non-redundant putative silencer and enhancer sequences, respectively. The CNN models identified 219,971 T cell silencers and 156,451 T cell enhancers from these sequences.

In the K562 cell line, experimentally validated silencers were used to evaluate the accuracy of the silencer prediction model. Those silencers were acquired from two resources: 3,796 K562 silencers identified with self-transcribing active regulatory region sequencing platform (STARRseq) (Doni Jayavelu et al. 2020) and 3,909 K562 silencers reported using the repressive ability of silencer element assay (ReSE) (Pang and Snyder 2020). After redundancy exclusion, we assembled 7,701 distinct silencers experimentally validated in K562 cells. To apply the CNN model in which the input is 1,000 bp sequences on these silencers, we extended these silencers into 1,000 bp genomic sequences centered at the midpoint of tested segments. To prevent a bias from prior knowledge on the estimate of the model accuracy, we excluded all 1,406 experimentally validated silencers that overlap training or validation sequences (either for building the SVM or the CNN model) from the computational model evaluation. 
In addition, the results of Sharpr-MPRA were used to evaluate silencer/enhancer predictions (Ernst et al. 2016). A low Sharpr-MPRA MaxPos score represents a negative regulatory element. In this study, a genomic sequence was assigned with a MaxPos score only when this sequence hosted the whole corresponding Sharpr-MPRA sequence.

\section{Enhancer-silencer transitions during development}

To identify enhancer-silencer transitions during development, we compared silencer profiles in mature cell types with the enhancer map in H1 hESCs. A T cell silencer overlapping an H1 hESC enhancer by over 600 bps was considered a DFRE. During embryonic development, the differentiation of T cells is a stepwise process from ESCs to mesodermal cells, HPCs, common lymphoid progenitors, and finally T cells (Kumar et al. 2018). In adulthood, T cells are differentiated from HPCs.

\section{Evaluating the repressive effect of mutations}

To evaluate the impact of silencer mutations, we focused on $y s$ and $y e$ in a built CNN model (Fig. 1A) as they predict the capability of genomic sequences being silencers and activators, respectively. Given the wild type (WT) and mutant allele (MU) of a mutation, the difference of $y s$ and $y e$ between these alleles directly measure the alternation of activating and repressing power of the host sequence. That is, the CNN-based silencing alteration score (CNNSAS) of a given mutation is defined as

$$
C N N-S A S=\left(y s_{W T}-y s_{M U}\right)-\left(y e_{W T}-y e_{M U}\right)
$$

where $y s_{W T}$ and $y s_{M U}$ are the probabilities of silencer activity in the WT and the MU sequences, respectively. $y e_{W T}$ and $y e_{M U}$ are the probabilities of enhancer activities of the corresponding sequences. In addition, we measured the silencing alteration caused by a mutation with the odd- 
ratios of $\left(y s_{W T}-y e_{W T}\right)$ to $\left(y s_{M U}-y e_{M U}\right)$ after deriving the probability function of $(y s-$ $y e$ ) and noticed the similar performance between $C N N-S A S$ scores and odd-ratio scores ("CNN-based odds ratios of mutations" in the Supplementary Notes and Fig. S12).

The $C N N-S A S$ distribution of all possible single nucleotide silencer mutations forms a T distribution with $69 \%$ of mutations having an absolute $C N N-S A S$ value smaller than 0.01 (Fig. S15). Using this distribution as a background, we evaluated the significance of observed $C N N$-SAS scores. A low value of the probability represents a mutation having a strong impact on the activity of the host silencer. A $C N N-S A S$ score was considered significant if this probability was less than $1 \%$.

\section{Data and tools used for analysis}

We downloaded the GWAS SNPs curated in the National Human Genome Research Institute (NHGRI) catalog as of January 2019 (McMahon et al. 2018). There were 70,578 unique SNPs in the catalog at that time. To account for the fact that GWAS SNPs might not be directly responsible for phenotypic alterations but might be in linkage disequilibrium (LD) with untested causal genetic variants (Cantor et al. 2010), we further expanded the SNP set by adding SNPs in a strong LD $\left(r^{2}>0.8\right)$ block with GWAS SNPs in at least one population from the 1000 Genomes Project. To that end, we acquired 1,409,462 GWAS SNPs associated with 2,722 traits. SNPs exhibiting a strong phenotypic influence are likely to be associated with multiple traits according to independent studies (Zhou et al. 2018). To account for this, GWAS SNPs were linearly weighted by the number of the traits linked to them when we evaluated the GWAS SNP density. The GWAS traits were identified as immunity-related when they contain keywords such 
as allergy, arthritis, asthma, Graves' disease, infection, inflammation, lupus, multiple sclerosis, Sjogren syndrome, type I diabetes mellitus, etc.

Whole-blood expression quantitative trait loci (eQTLs) used in this study were downloaded from the Genotype-Tissue Expression Project, GTEx database version 8 (The GTEx Consortium 2015). To further evaluate the tissue-specificity of the predicted DFREs, we collected eQTLs detected in T cells from the Blueprint epigenome project (Chen et al. 2016). EQTLs in iPSC were download from DeBoeve's study (DeBoever et al. 2017).

We downloaded 14,183 HepG2 reporter assay quantitative trait loci (raQTLs) from the study (van Arensbergen et al. 2019). These SNPs are associated with a significant change in reporter gene activity in HepG2 cells. We also downloaded additional 14,183 SNPs which correspond to an insignificant change in the expression of reporter genes (namely, non-raQTL mutations) as analysis background.

We used the Genomic Regions Enrichment of Annotation Tool (GREAT) (McLean et al. 2010) to evaluate Gene Ontology (GO) biological processes and molecular functions associated with different RE groups. The element-gene association setting was selected as "two nearest genes."

We explored conserved elements predicted using the 46-ways placental phastCons scores (Siepel et al. 2005). A notable overlap of a genome sequence with conserved elements is indicative of an evolutionary constraint imposed on that sequence. The density of SNPs in genomic sequences was used to assess the evolutionary pressure acting on genomic segments in the human lineage. A low SNP density corresponds to strong selective pressure. The SNPs reported in the 1,000 Genomes Project (The Genomes Project et al. 2015) were employed for this analysis. 
HESC TAD boundaries were downloaded from the Topologically Associating Domain Knowledge Base (TADKB) database in which the boundaries, in the resolution of $10 \mathrm{~kb}$, were detected by using the Gaussian Mixture model and Proportion test (Liu et al. 2019). The chromatin contacts in hESC were downloaded from the database assembled in the Jung's study (Jung et al. 2019). The TADs and genomic contacts in T cells were downloaded from the Yang's report (Yang et al. 2020). We used the detections for unstimulated T cells. The TAD boundaries were defined as the $10 \mathrm{~kb}$-long genomic regions centering at all the ends of TADs.

\section{Software availability}

The custom python scripts (training CNN models and predicting enhancers/silencers with a built CNN model) are available on GitHub (https://github.com/ncbi/SilencerEnhancerPredict) and in Supplemental Code (Supplemental_code.zip).

\section{Competing interest statement}

The authors declare no competing interests.

\section{Acknowledgments}

This research was supported by the Intramural Research Program of the National Library of Medicine and the National Human Genome Research Institute, National Institutes of Health. We are grateful to Dorothy L. Buchhagen and Chris Hill and Sanjarbek Hudaiberdiev and Wei Song for critical reading of the manuscript.

\section{Author contributions}


D.H. performed computational analysis and analyzed the data. I.O. supervised computational work. D.H. prepared figures and tables. D.H. and I.O. wrote the manuscript.

\section{References}

Alipanahi B, Delong A, Weirauch MT, Frey BJ. 2015. Predicting the sequence specificities of DNA- and RNA-binding proteins by deep learning. Nature Biotechnology 33: 831-838.

Benton ML, Talipineni SC, Kostka D, Capra JA. 2019. Genome-wide enhancer annotations differ significantly in genomic distribution, evolution, and function. BMC Genomics 20: 511.

Berest I, Arnold C, Reyes-Palomares A, Palla G, Rasmussen KD, Giles H, Bruch P-M, Huber W, Dietrich S, Helin K et al. 2019. Quantification of Differential Transcription Factor Activity and Multiomics-Based Classification into Activators and Repressors: diffTF. Cell Reports 29: 3147-3159.e3112.

Bessis A, Champtiaux N, Chatelin L, Changeux J-P. 1997. The neuron-restrictive silencer element: A dual enhancer/silencer crucial for patterned expression of a nicotinic receptor gene in the $\square$ brain. Proceedings of the National Academy of Sciences 94: 5906.

Brand AH, Breeden L, Abraham J, Sternglanz R, Nasmyth K. 1985. Characterization of a "silencer" in yeast: A DNA sequence with properties opposite to those of a transcriptional enhancer. Cell 41: 41-48.

Cai Y, Zhang Y, Loh YP, Tng JQ, Lim MC, Cao Z, Raju A, Lieberman Aiden E, Li S, Manikandan L et al. 2021. H3K27me3-rich genomic regions can function as silencers to repress gene expression via chromatin interactions. Nature Communications 12: 719.

Cantor RM, Lange K, Sinsheimer JS. 2010. Prioritizing GWAS results: A review of statistical methods and recommendations for their application. Am J Hum Genet 86: 6-22.

Chen L, Ge B, Casale FP, Vasquez L, Kwan T, Garrido-Martín D, Watt S, Yan Y, Kundu K, Ecker S et al. 2016. Genetic Drivers of Epigenetic and Transcriptional Variation in Human Immune Cells. Cell 167: 1398-1414.e1324.

Chen S, Yang J, Wei Y, Wei X. 2020. Epigenetic regulation of macrophages: from homeostasis maintenance to host defense. Cellular \& Molecular Immunology 17: 36-49.

DeBoever C, Li H, Jakubosky D, Benaglio P, Reyna J, Olson KM, Huang H, Biggs W, Sandoval E, D'Antonio M et al. 2017. Large-Scale Profiling Reveals the Influence of Genetic Variation on Gene Expression in Human Induced Pluripotent Stem Cells. Cell Stem Cell 20: 533-546.e537.

Della Rosa M, Spivakov M. 2020. Silencers in the spotlight. Nature Genetics 52: 244-245.

Dixon JR, Jung I, Selvaraj S, Shen Y, Antosiewicz-Bourget JE, Lee AY, Ye Z, Kim A, Rajagopal N, Xie W et al. 2015. Chromatin architecture reorganization during stem cell differentiation. Nature 518: 331-336.

Doni Jayavelu N, Jajodia A, Mishra A, Hawkins RD. 2020. Candidate silencer elements for the human and mouse genomes. Nature Communications 11: 1061.

Erceg J, Pakozdi T, Marco-Ferreres R, Ghavi-Helm Y, Girardot C, Bracken AP, Furlong EEM. 2017. Dual functionality of cis-regulatory elements as developmental enhancers and Polycomb response elements. Genes \& development 31: 590-602. 
Ernst J, Kellis M. 2017. Chromatin-state discovery and genome annotation with ChromHMM. Nature Protocols 12: 2478-2492.

Ernst J, Melnikov A, Zhang X, Wang L, Rogov P, Mikkelsen TS, Kellis M. 2016. Genome-scale high-resolution mapping of activating and repressive nucleotides in regulatory regions. Nature Biotechnology 34: 1180.

Gao T, Qian J. 2020. EnhancerAtlas 2.0: an updated resource with enhancer annotation in 586 tissue/cell types across nine species. Nucleic Acids Research 48: D58-D64.

Gisselbrecht SS, Palagi A, Kurland JV, Rogers JM, Ozadam H, Zhan Y, Dekker J, Bulyk ML. 2020. Transcriptional Silencers in Drosophila Serve a Dual Role as Transcriptional Enhancers in Alternate Cellular Contexts. Molecular Cell 77: 324-337.e328.

Grinberg-Bleyer Y, Caron R, Seeley JJ, De Silva NS, Schindler CW, Hayden MS, Klein U, Ghosh S. 2018. The Alternative NF-кB Pathway in Regulatory T Cell Homeostasis and Suppressive Function. J Immunol 200: 2362-2371.

Györy I, Boller S, Nechanitzky R, Mandel E, Pott S, Liu E, Grosschedl R. 2012. Transcription factor Ebf1 regulates differentiation stage-specific signaling, proliferation, and survival of B cells. Genes \& development 26: 668-682.

Halfon MS. 2020. Silencers, Enhancers, and the Multifunctional Regulatory Genome. Trends in Genetics 36: 149-151.

Harley JB, Alarcón-Riquelme ME, Criswell LA, Jacob CO, Kimberly RP, Moser KL, Tsao BP, Vyse TJ, Langefeld CD, Nath SK et al. 2008. Genome-wide association scan in women with systemic lupus erythematosus identifies susceptibility variants in ITGAM, PXK, KIAA1542 and other loci. Nat Genet 40: 204-210.

Huang D, Petrykowska HM, Miller BF, Elnitski L, Ovcharenko I. 2019. Identification of human silencers by correlating cross-tissue epigenetic profiles and gene expression. Genome Research.

Jacob F, Monod J. 1961. Genetic regulatory mechanisms in the synthesis of proteins. Journal of Molecular Biology 3: 318-356.

Johnson WC, Ordway AJ, Watada M, Pruitt JN, Williams TM, Rebeiz M. 2015. Genetic Changes to a Transcriptional Silencer Element Confers Phenotypic Diversity within and between Drosophila Species. PLOS Genetics 11: e1005279.

Jung I, Schmitt A, Diao Y, Lee AJ, Liu T, Yang D, Tan C, Eom J, Chan M, Chee S et al. 2019. A compendium of promoter-centered long-range chromatin interactions in the human genome. Nature genetics 51: 1442-1449.

Kang JW, Kim Y, Lee Y, Myung K, Kim YH, Oh CK. 2021. AML poor prognosis factor, TPD52, is associated with the maintenance of haematopoietic stem cells through regulation of cell proliferation. J Cell Biochem 122: 403-412.

Kehayova P, Monahan K, Chen W, Maniatis T. 2011. Regulatory elements required for the activation and repression of the protocadherin- $\alpha$ gene cluster. Proceedings of the National Academy of Sciences 108: 17195.

Koh FM, Lizama CO, Wong P, Hawkins JS, Zovein AC, Ramalho-Santos M. 2015. Emergence of hematopoietic stem and progenitor cells involves a Chd1-dependent increase in total nascent transcription. Proceedings of the National Academy of Sciences 112: E1734.

Koo PK, Ploenzke M. 2020. Deep learning for inferring transcription factor binding sites. Current Opinion in Systems Biology 19: 16-23.

Kumar BV, Connors TJ, Farber DL. 2018. Human T Cell Development, Localization, and Function throughout Life. Immunity 48: 202-213. 
Lefebvre V. 2010. The SoxD transcription factors--Sox5, Sox6, and Sox13--are key cell fate modulators. The international journal of biochemistry \& cell biology 42: 429-432.

Liu T, Porter J, Zhao C, Zhu H, Wang N, Sun Z, Mo Y-Y, Wang Z. 2019. TADKB: Family classification and a knowledge base of topologically associating domains. BMC Genomics 20: 217.

Mayes MD, Bossini-Castillo L, Gorlova O, Martin JE, Zhou X, Chen WV, Assassi S, Ying J, Tan FK, Arnett FC et al. 2014. Immunochip analysis identifies multiple susceptibility loci for systemic sclerosis. Am J Hum Genet 94: 47-61.

McLean CY, Bristor D, Hiller M, Clarke SL, Schaar BT, Lowe CB, Wenger AM, Bejerano G. 2010. GREAT improves functional interpretation of cis-regulatory regions. Nature Biotechnology 28: 495-501.

McMahon A, Malangone C, Suveges D, Sollis E, Cunningham F, Riat HS, MacArthur JA L, Hayhurst J, Morales J, Guillen JA et al. 2018. The NHGRI-EBI GWAS Catalog of published genome-wide association studies, targeted arrays and summary statistics 2019 . Nucleic Acids Research 47: D1005-D1012.

Ngan CY, Wong CH, Tjong H, Wang W, Goldfeder RL, Choi C, He H, Gong L, Lin J, Urban B et al. 2020. Chromatin interaction analyses elucidate the roles of PRC2-bound silencers in mouse development. Nature Genetics 52: 264-272.

Ogiyama Y, Schuettengruber B, Papadopoulos GL, Chang J-M, Cavalli G. 2018. PolycombDependent Chromatin Looping Contributes to Gene Silencing during Drosophila Development. Molecular Cell 71: 73-88.e75.

Ong C-T, Corces VG. 2014. CTCF: an architectural protein bridging genome topology and function. Nature reviews Genetics 15: 234-246.

Pang B, Snyder MP. 2020. Systematic identification of silencers in human cells. Nature Genetics 52: 254-263.

Roadmap Epigenomics Consortium, Kundaje A, Meuleman W, Ernst J BM, Yen A, Kheradpour P, Wang J, Whitaker JW, Schultz MD, Ward LD et al. 2015. Integrative analysis of 111 reference human epigenomes. Nature 518: 317-330.

Rojano E, Seoane P, Ranea JAG, Perkins JR. 2018. Regulatory variants: from detection to predicting impact. Briefings in Bioinformatics 20: 1639-1654.

Senftleben U, Cao Y, Xiao G, Greten Florian R, Krähn G, Bonizzi G, Chen Y, Hu Y, Fong A, Sun S-C et al. 2001. Activation by IKK $\alpha$ of a Second, Evolutionary Conserved, NF- $\kappa$ B Signaling Pathway. Science 293: 1495-1499.

Siepel A, Bejerano G, Pedersen JS, Hinrichs AS, Hou M, Rosenbloom K, Clawson H, Spieth J, Hillier LW, Richards S et al. 2005. Evolutionarily conserved elements in vertebrate, insect, worm, and yeast genomes. Genome Research 15: 1034-1050.

Stathopoulos A, Levine M. 2005. Localized repressors delineate the neurogenic ectoderm in the early Drosophila embryo. Dev Biol 280: 482-493.

Tartey S, Takeuchi O. 2015. Chromatin Remodeling and Transcriptional Control in Innate Immunity: Emergence of Akirin2 as a Novel Player. Biomolecules 5: 1618-1633.

The Genomes Project C Auton A Abecasis GR Altshuler DM Durbin RM Abecasis GR Bentley DR Chakravarti A Clark AG Donnelly P et al. 2015. A global reference for human genetic variation. Nature 526: 68 .

The GTEx Consortium. 2015. The Genotype-Tissue Expression (GTEx) pilot analysis: Multitissue gene regulation in humans. Science 348: 648-660. 
Thibodeau A, Uyar A, Khetan S, Stitzel ML, Ucar D. 2018. A neural network based model effectively predicts enhancers from clinical ATAC-seq samples. Scientific Reports 8: 16048.

van Arensbergen J, Pagie L, FitzPatrick VD, de Haas M, Baltissen MP, Comoglio F, van der Weide RH, Teunissen H, Võsa U, Franke L et al. 2019. High-throughput identification of human SNPs affecting regulatory element activity. Nature genetics 51: 1160-1169.

Wang P, Deng Y, Yan X, Zhu J, Yin Y, Shu Y, Bai D, Zhang S, Xu H, Lu X. 2020. The Role of ARID5B in Acute Lymphoblastic Leukemia and Beyond. Front Genet 11: 598-598.

Yang J, McGovern A, Martin P, Duffus K, Ge X, Zarrineh P, Morris AP, Adamson A, Fraser P, Rattray $M$ et al. 2020. Analysis of chromatin organization and gene expression in T cells identifies functional genes for rheumatoid arthritis. Nature Communications 11: 4402.

Zhou J, Theesfeld CL, Yao K, Chen KM, Wong AK, Troyanskaya OG. 2018. Deep learning sequence-based $\mathrm{ab}$ initio prediction of variant effects on expression and disease risk. Nature Genetics 50: 1171-1179.

Zhou J, Troyanskaya OG. 2015. Predicting effects of noncoding variants with deep learningbased sequence model. Nature Methods 12: 931-934.

\section{Figure Legends}

Figure 1. A deep learning model to predict silencers. (A) A schematic of the deep learning model used to predict cell type-specific silencers and enhancers. The number of kernels or neurons in a layer is listed in parentheses. The input of the model consists of $1 \mathrm{~kb}$ genomic sequences, and the output is a set of three probabilities of the input sequence being a silencer (ys), an enhancer (ye), or a non-functional sequence (yn). (B) ROCs and PRCs of the prediction models in three human cell types. The results in the additional three cell lines are presented in Fig. S1. (C) ROC and PRC classification accuracy in prediction of ReSE (Pang and Snyder 2020) and MPRA (Doni Jayavelu et al. 2020) experimentally characterized silencers. (D) SharprMPRA MaxPos scores in K562 cells. EN and SL are the predicted enhancers and silencers, respectively. NonEN represents the DNase-seq peaks that overlap with H3K27ac ChIP-seq peaks but have not been predicted as enhancers in this study. NonSL represents the H3K27me3 ChIPseq peaks not predicted as silencers, while the DHS column corresponds to all DNase-seq peaks. The count under a group label (X-axis) is the size of the corresponding sequence set. (E) The density of GWAS SNPs in the predicted silencers (SL), the sequences with H3K27me3 peaks but no H3K27ac peaks (H3K27me3/-H3K27ac), and the predicted enhancers (EN). The background consists of the genomic sequences having the GC content and repeat density matching to $\mathrm{T}$ cell SLs. $* *-p<10^{-10}$. White asterisks are the significance enrichments of GWAS SNPs as 
compared to $\mathrm{H} 3 \mathrm{~K} 27 \mathrm{me} 3 /-\mathrm{H} 3 \mathrm{~K} 27 \mathrm{ac}$, and black asterisks are the significant enrichments in enhancers as compared to silencers.

Figure 2. DFREs are consequential. (A) A distribution pie chart of the silencers (SLrs) and DFREs for primary T cells in peripheral blood. (B) Evolutionary sequence conservation of the DFREs (red), SLrs (grey), and ENrs (orange) computed using the overlap with conserved segments and the density of common SNPs. The asterisks above the bars indicate the significant enrichments as compared to the background consisting of a group of the randomly sampled genomic regions with the GC and repeat contents matching the silencers. $* *-p<10^{-10}$. (C) Molecular functions significantly associated with the DFREs. The results are from the web tool GREAT using all the silencers as background. (D) Enrichment of DFREs in the loci of the TFs and $\mathrm{CM}$ genes. $* *-p<10^{-5}$. White asterisks in the bars represent the significant enrichment levels as compared to the SLrs. The presented p-values are the enrichments as compared to all TF and CMs. (E) Enrichment of GWAS SNPs and eQTLs in DFREs, SLrs, and ENrs. The density of SNPs per kb is listed in the bars. The asterisks (and the values) next to the bars quantify the significance of enrichment as compared to the background. $* *-p<10^{-5}$. (F) GWAS traits with which the associated SNPs are enriched in the DFREs as compared with ENrs. Only the GWAS traits significantly enriched in the DFREs are presented. The azure diamonds highlight immunity-related traits. Red and black dots on the top indicate the significant enrichments $(p<0.05)$ of GWAS SNPs in DFREs and SLrs, respectively. The details about these results are listed in Table $\mathrm{S} 2$.

Figure 3. Enrichment of significant CNN-SAS scores in the DFREs. (A) Correlation between CNN-SASs and raQTL scores on the raQTL SNPs (blue dots) and non raQTL SNPs (grey dots). (B) Distribution of raQTL scores in mutation groups binned based on CNN-SASs. The number under each box is the fraction of negative raQTL scores. A negative raQTL score represents the increase in silencing activity. $* *-p<10^{-10}$ and $*-p<10^{-2}$ represent the enrichment significance in negative raQTL scores as compared to all tested mutations. (C) Correlation between CNN-SAS scores and eQTL scores in T cells. (D) Fraction of significant CNN-SASs across GWAS and eQTL sets of SNPs. (E) The CNN-SAS scores of the SNPs in a LD block associated with systemic lupus erythematosus and systemic sclerosis. Rs12631656, a DFRE SNP, 
is the only one having a significant CNN-SAS score. The table lists the mapping significance levels (i.e., FIMO significance $p$ values) of the ARID5B and SOX13 binding motifs for the sequences carrying the reference or alternative allele at rs 12631656.

Figure 4. Distinct binding syntax of the DFREs. Enrichment of ChIP-seq TFBSs in (A) T cells and (B) hESCs. The heatmap on top illustrates enrichment/depletion significance $\left(-\log _{10}\right.$ binomial test p) in DFREs, SLrs, and ENrs as compared to all DNase-seq and H3K27me3 ChIPseq peaks in the corresponding cell types. The red and blue shades represent the significance levels of enrichment and depletion, respectively. (C) Functional specificity of silencer and enhancer TFBSs in these DFREs. According to their activity in T cells and H1 hESCs, these TFBSs are categorized into two groups: function-specific (i.e., unique to one cell type) and shared (i.e., shared by two cell types). The numbers in the bars are the average numbers of TFBSs per DFRE. (D) Distribution of silencer TFBSs (the blue bars and line) and enhancer TFBSs (the orange bars and line) in the DFREs. (E) Distance of silencer TFBSs to their nearest enhancer TFBSs. The background distribution was generated through randomly scattering silencer and enhancer TFBSs within the DFREs.

Figure 5. Characterization of the DFREs detected in HPCs. (A) Fraction of HPC silencers working as DFREs. (B) Evolutionary sequence conservation measured using the overlap with the conserved segments (left panel) and common SNP density (right panel) of HPC DFREs, SLrs, and ENrs. The background in Fig. 2 is also included here (represented by "background") for consistency. (C) Enrichment of GWAS SNPs and eQTLs in the HPC. The numbers of SNPs per $\mathrm{kb}$ are listed in the bars. In (B-C), the asterisks above the bars represent the significance levels as compared to the background. (D) Developmental specificity of DFREs, SLr, and enhancers in HPCs. The numbers in bars are the numbers of REs. Here, "shared" represents the REs shared in HPCs and T cells, while "specific" is for the REs acting in HPCs but not in T cells. $* *-p<$ $10^{-5}$. 
A

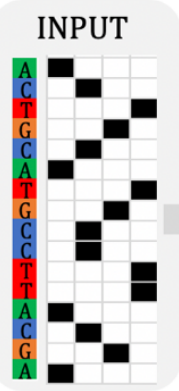

B

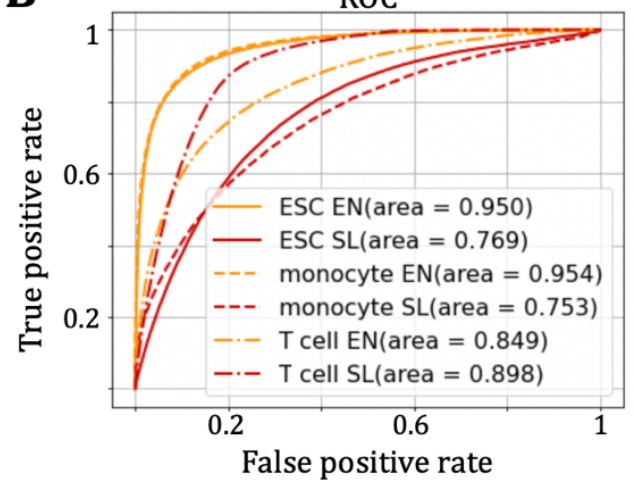

C

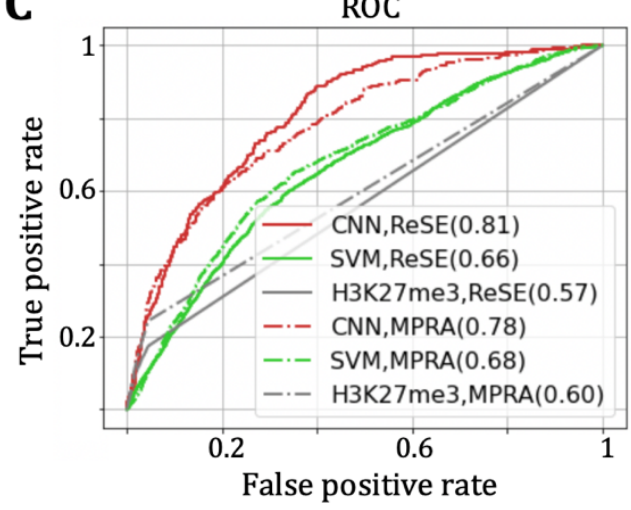

CNN

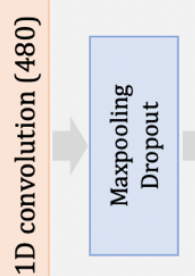

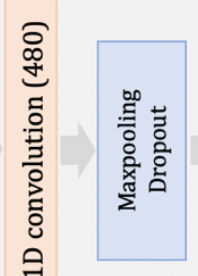

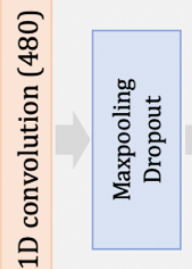

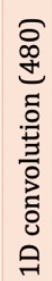

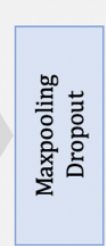

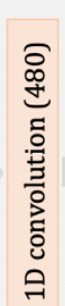

OUTPUT

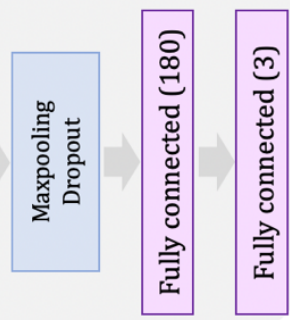

ys

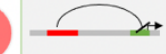

yn

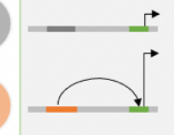

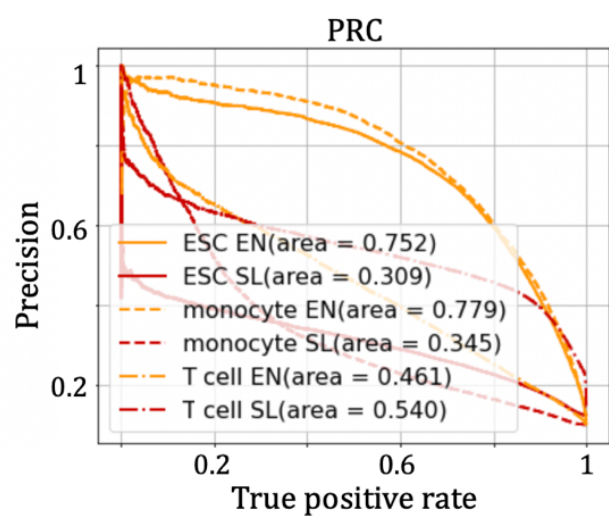

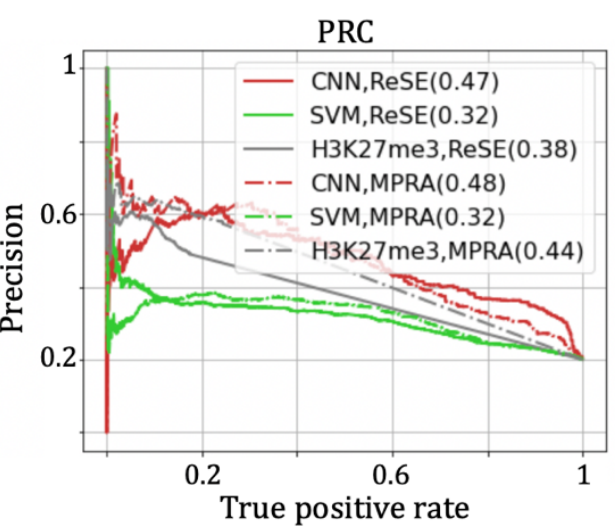

$\mathbf{D}_{0}$

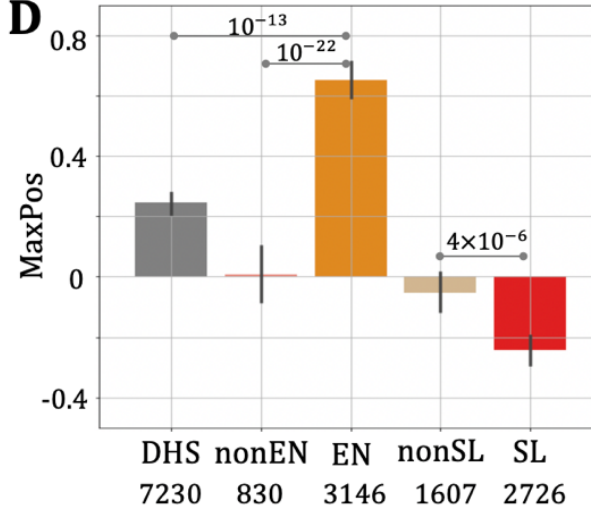

E

$\varpi \mathrm{SL}=\mathrm{H} 3 \mathrm{~K} 27 \mathrm{me} /-\mathrm{H} 2 \mathrm{~K} 27 \mathrm{ac}=\mathrm{EN}$

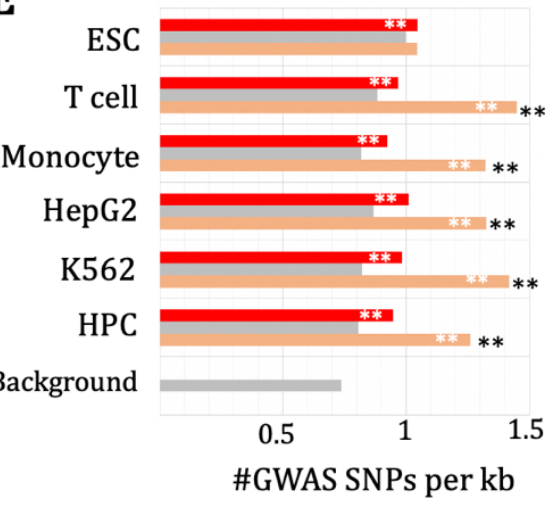




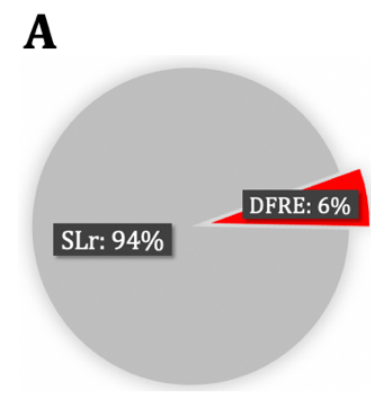

C

B

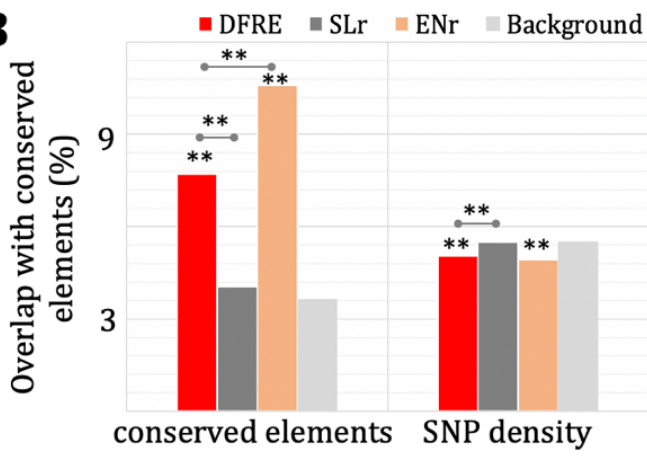

$-\log _{10}$ (hypergeometric $p$ value, DFREs vs SLs)

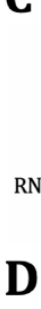

Dop 1000 highly expressed

Top 2000 highly expressed

$\mathrm{TF} / \mathrm{CMs}$

All TF/CMs

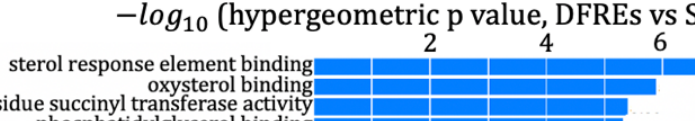

hydrolipoyllysin-residue succinyl transferase activity

\begin{tabular}{l|l|l|}
\hline phosphatidylglycerol binding &
\end{tabular}

phosphatidylglycerol binding
II type 3 promoter DNA binding

FK605 binding
CD8 receptor binding

Arp $2 / 3$ complex binding

Arp cell receptor binding

Enrichment
E Enrichment of GWAS or eQTL SNPs in SLS
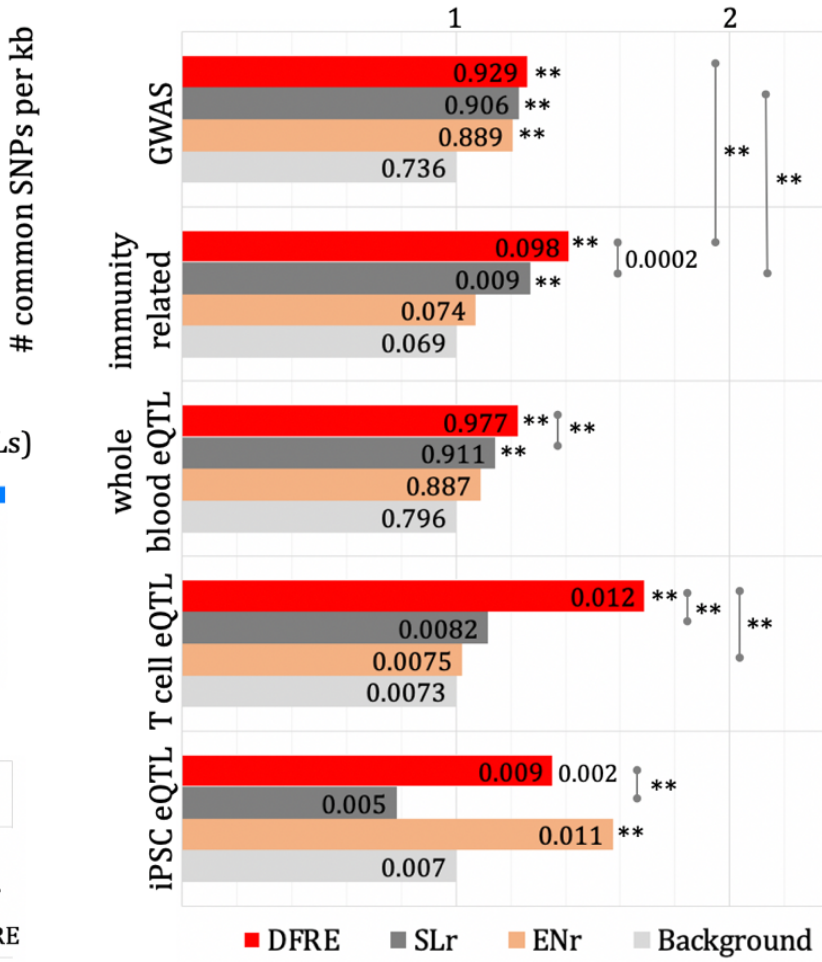

\section{$\mathbf{F}$}

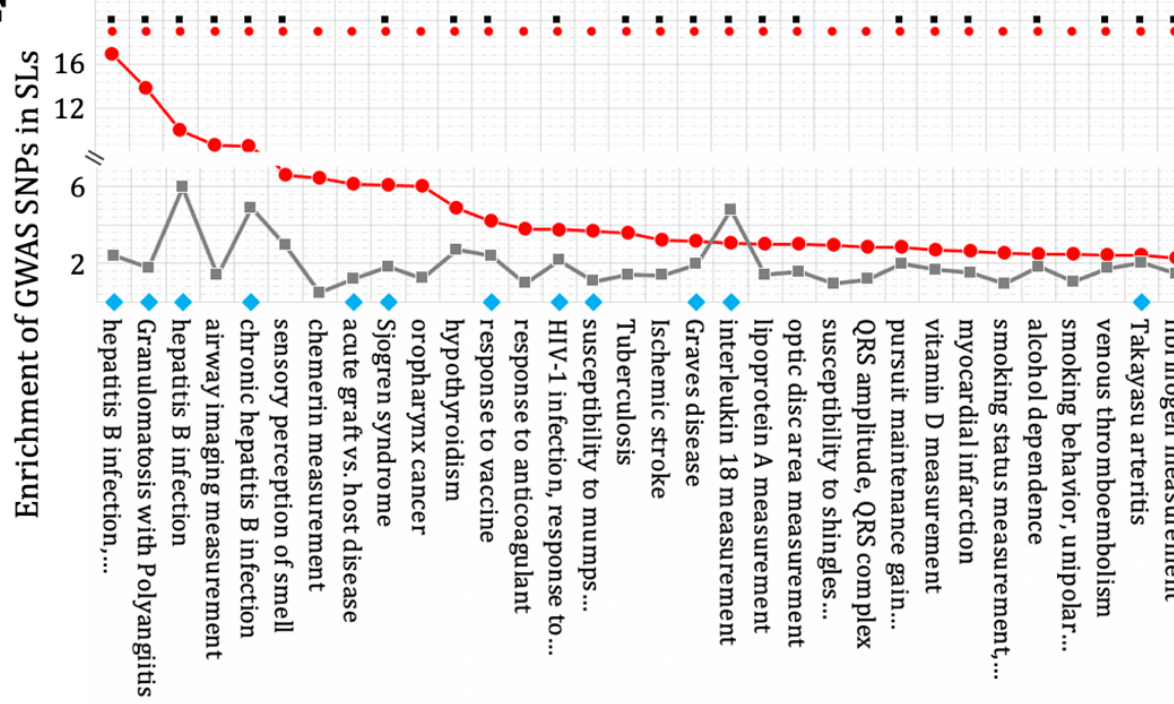

GWAS traits 

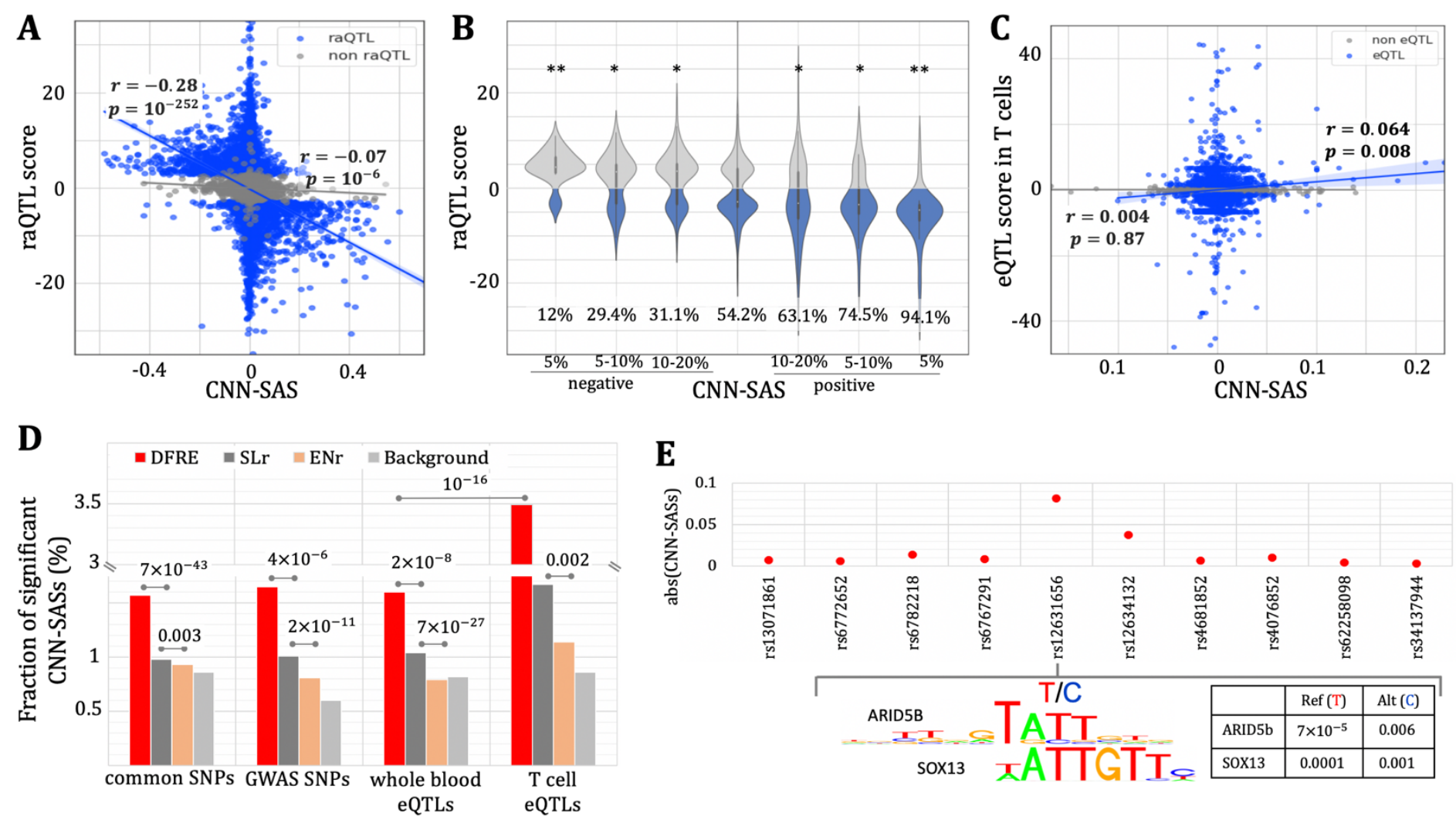

E

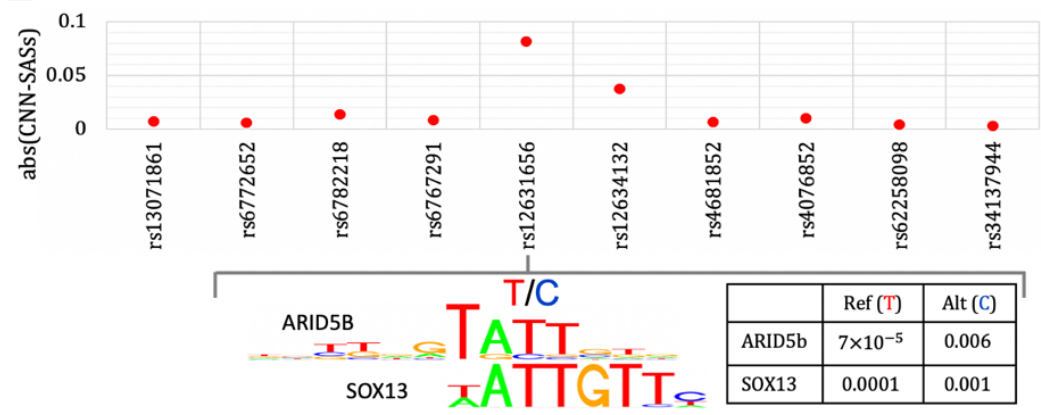




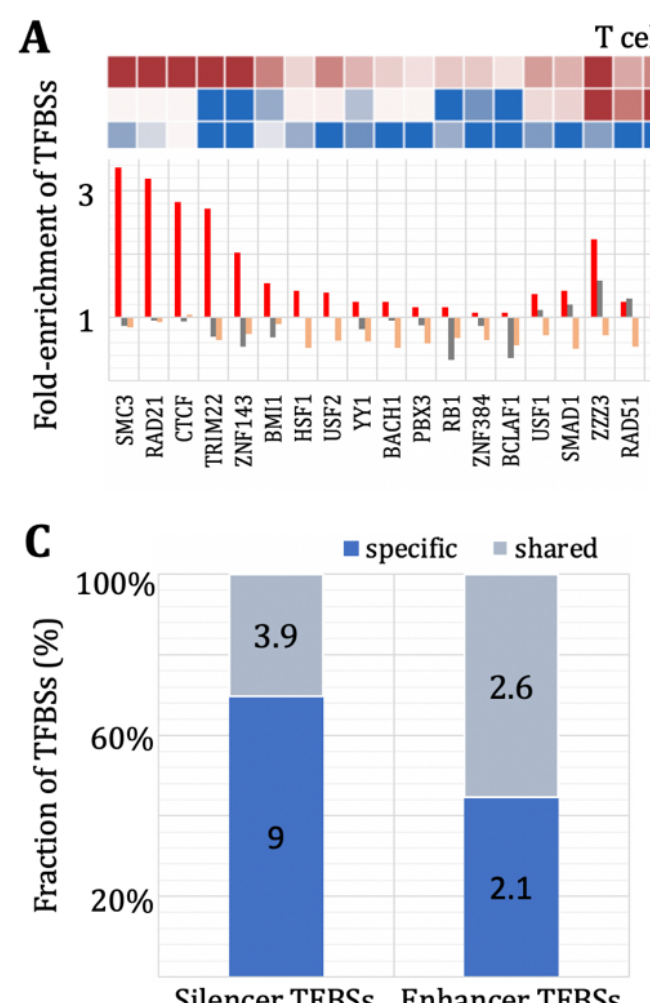

cells

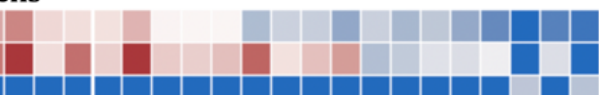

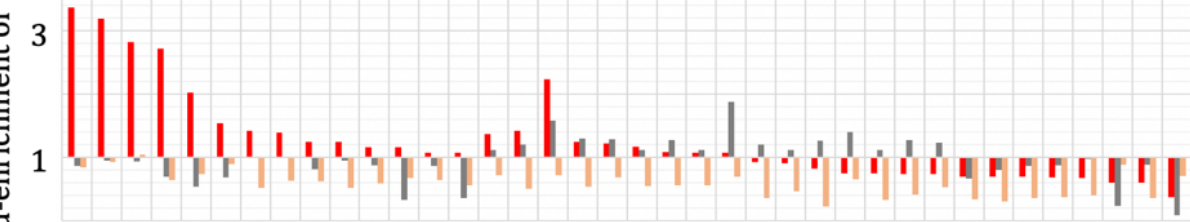

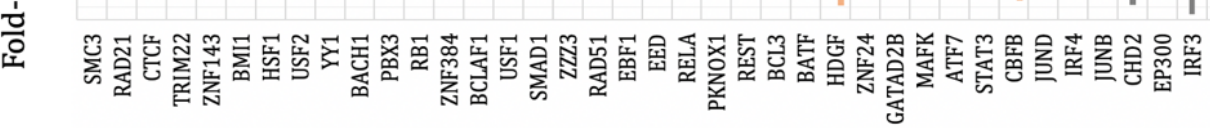

C

Silencer TFBSs Enhancer TFBSs
D

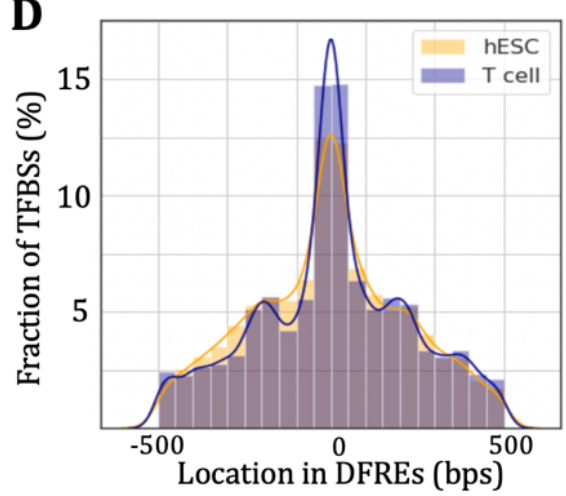

B

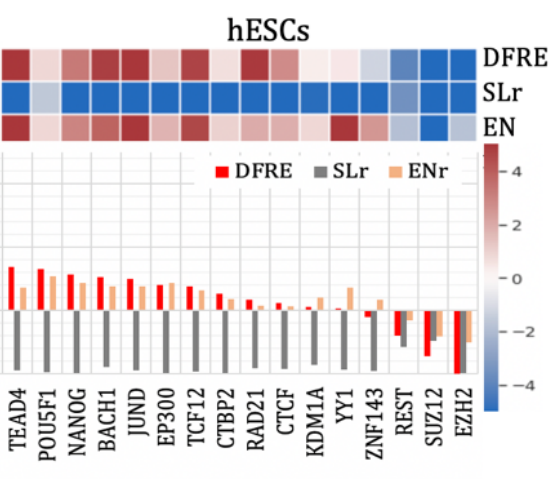

E

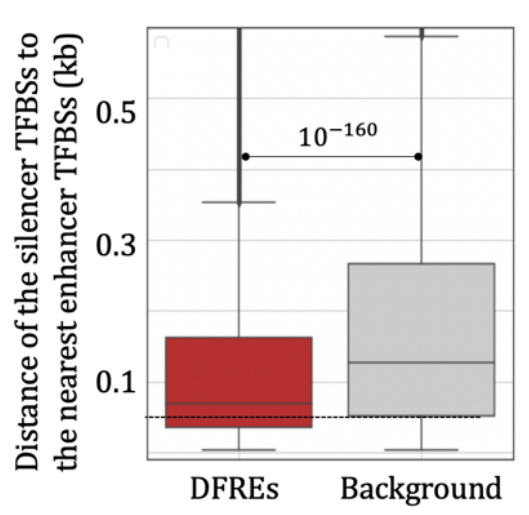


A

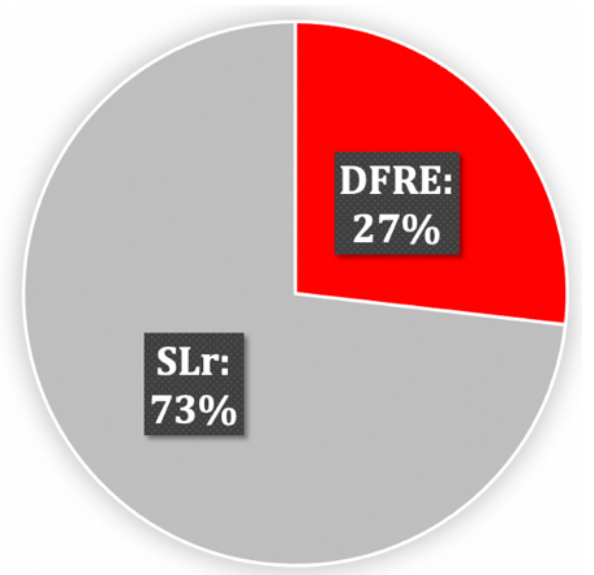

C

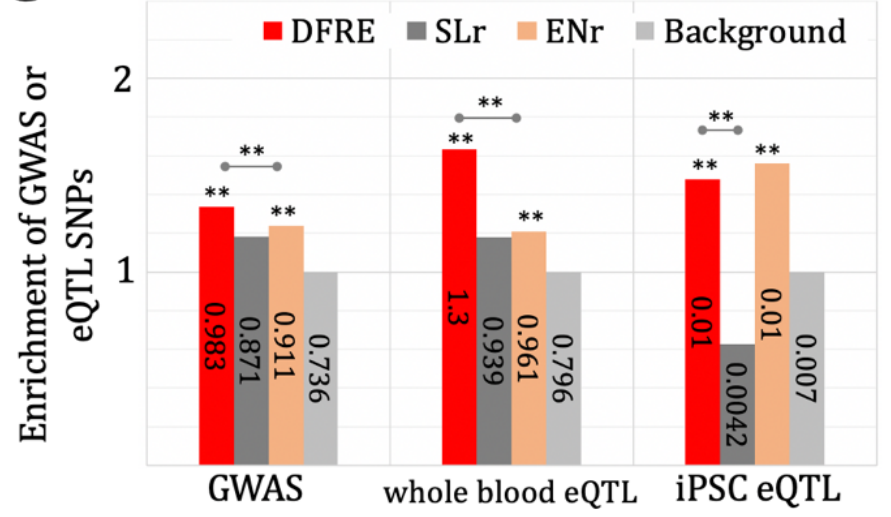

B

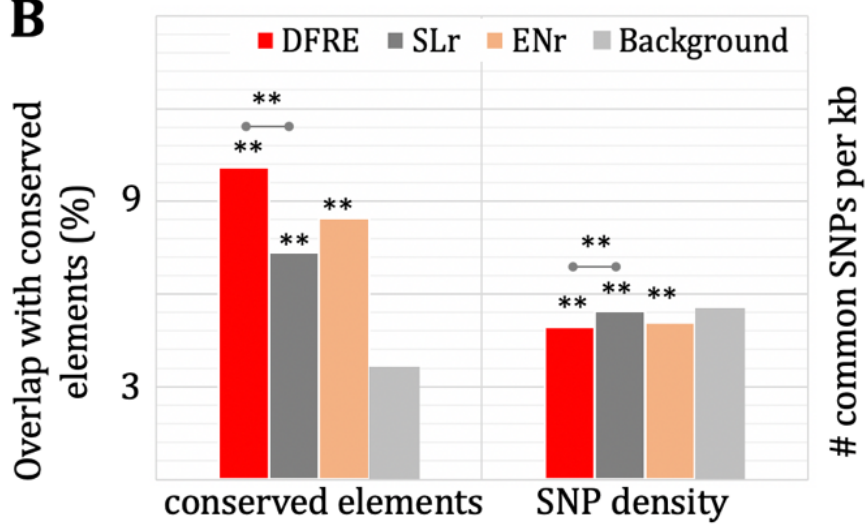

D

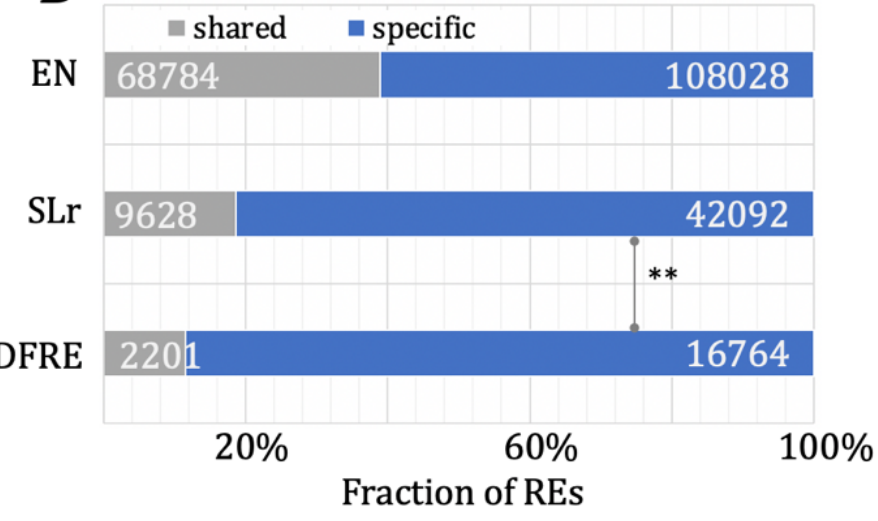




\section{Corrigenda}

Genome Research 32: 778-790 (2022)

\section{Corrigendum: An association test of the spatial distribution of rare missense variants within protein structures identifies Alzheimer's disease-related patterns}

Bowen Jin, John A. Capra, Penelope Benchek, Nicholas Wheeler, Adam C. Naj, Kara L. Hamilton-Nelson, John J. Farrell, Yuk Yee Leung, Brian Kunkle, Badri Vadarajan, Gerard D. Schellenberg, Richard Mayeux, Li-San Wang, Lindsay A. Farrer, Margaret A. Pericak-Vance, Eden R. Martin, Jonathan L. Haines, Dana C. Crawford, and William S. Bush

In the above-mentioned article, the authors would like to correct errors related to Figures 5 and 6 . Figure 6 was a duplication of Figure 5, and the variant positions within Figure 5 were incorrect due to an inconsistency in the residue numbering of the PDB:6XDS with its corresponding UniProt entry. This error was isolated to the 6XDS structure only, and did not qualitatively change the results or conclusions of the analysis. Both Figures 5 and 6 have been updated in the revised article online.

The authors apologize for any confusion this might have caused.

doi: $10.1101 /$ gr. 276900.122

Genome Research 32: 437-448 (2022)

\section{Corrigendum: Enhancer-silencer transitions in the human genome Di Huang and Ivan Ovcharenko}

In the above-mentioned article, the authors would like to correct the citation of the prior studies of mesodermal silencers functioning as enhancers during Drosophila embryonic development and provide additional background information in the introductory section. Although the original citation was factually correct, the expanded citation provides a more accurate and extended description of the prior work on enhancersilencer transitions in Drosophila. This correction has no impact on either the data, results, or conclusions reported in this article.

The corrected text (third paragraph, fourth sentence) reads as follows:

"...whereas 22 out of 29 (76\%) identified mesodermal silencers were shown to act as DFREs in large enhancer screens (Gallo et al. 2011; Bonn et al. 2012) and were later augmented by additional six DFREs picked up by a targeted enhancer analysis (thus, bringing the total to 28/29 [Gisselbrecht et al. 2020])."

The additional references are as follows:

Bonn S, Zinzen RP, Girardot C, Gustafson EH, Perez-Gonzalez A, Delhomme N, Ghavi-Helm Y, Wilczyński B, Riddell A, Furlong EEM. 2012. Tissue-specific analysis of chromatin state identifies temporal signatures of enhancer activity during embryonic development. Nat Genet 44: 148-156. doi:10.1038/ ng.1064

Gallo SM, Gerrard DT, Miner D, Simich M, Des Soye B, Bergman CM, Halfon MS. 2011. REDfly v3.0: toward a comprehensive database of transcriptional regulatory elements in Drosophila. Nucleic Acids Res 39: D118-D123. doi:10.1093/nar/gkq999

These changes have been updated in the article online. The authors apologize for any inconvenience.

doi: $10.1101 /$ gr.276950.122 
Genome Research 32: 986-1003 (2022)

Corrigendum: Precise genotyping of circular mobile elements from metagenomic data uncovers human-associated plasmids with recent common ancestors

Nitan Shalon, David A. Relman, and Eitan Yaffe

The authors would like to correct the email of the corresponding author, Eitan Yaffe, to the following: eitan.yaffe@stanford.edu.

This correction has been updated in the revised article online.

doi: $10.1101 /$ gr.276946.122

Genome Research 32: 534-544 (2022)

Corrigendum: NicE-C efficiently reveals open chromatin-associated chromosome interactions at high resolution

Zhengyu Luo, Ran Zhang, Tengfei Hu, Yuting Zhu, Yueming Wu, Wenfei Li, Zhi Zhang, Xuebiao Yao, Haiyi Liang, and Xiaoyuan Song

The authors would like to correct the inadvertent omission of a funding source in the Acknowledgments section in the above-mentioned article. The corrected text is as follows and has been updated in the article online:

"This work was supported by the National Key Scientific Program of China (2016YFA0100502), the research project of the Joint Laboratory of University of Science and Technology of China and Anhui Mental Health Center (2019LH03), the Fundamental Research Funds for the Central Universities (WK2070210004), and China Postdoctoral Science Foundation (2021M703091)."

doi: 10.1101/gr.276968.122 


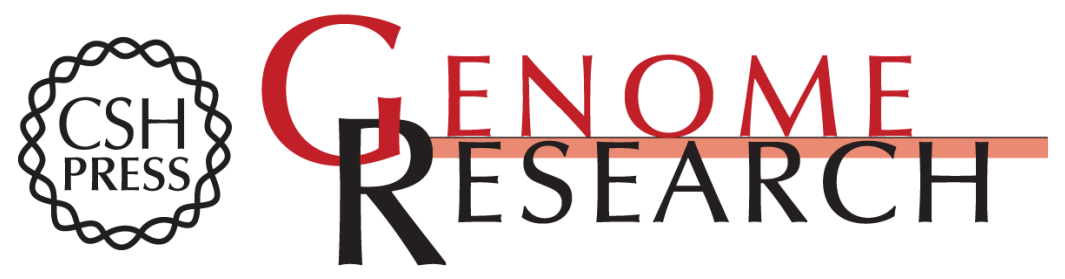

\section{Enhancer-silencer transitions in the human genome}

Di Huang and Ivan Ovcharenko

Genome Res. published online February 1, 2022

Access the most recent version at doi:10.1101/gr.275992.121

\section{Supplemental http://genome.cshlp.org/content/suppl/2022/02/21/gr.275992.121.DC1 Material}

Related Content Corrigendum: Enhancersilencer transitions in the human genome Di Huang and Ivan Ovcharenko

Genome Res. June , 2022 32: 1228_2

$\mathbf{P}<\mathbf{P} \quad$ Published online February 1, 2022 in advance of the print journal.

Accepted Peer-reviewed and accepted for publication but not copyedited or typeset; accepted Manuscript manuscript is likely to differ from the final, published version.

Open Access Freely available online through the Genome Research Open Access option.

License This is a work of the US Government.

Email Alerting Receive free email alerts when new articles cite this article - sign up in the box at the Service top right corner of the article or click here.

\section{Affordable, Accurate Sequencing.}

To subscribe to Genome Research go to:

https://genome.cshlp.org/subscriptions 